\title{
Development of Vacuum Vessel Design and Analysis Module for CFETR Integration Design Platform
}

\author{
Chen Zhu, ${ }^{1}$ Minyou Ye, ${ }^{1,2}$ Xufeng Liu, ${ }^{2}$ Shenji Wang, ${ }^{1}$ Shifeng Mao,, \\ Zhongwei Wang, ${ }^{2}$ and Yi Yu ${ }^{1}$ \\ ${ }^{1}$ School of Nuclear Science and Technology, University of Science and Technology of China, Hefei, China \\ ${ }^{2}$ Institute of Plasma Physics, Chinese Academy of Sciences, Hefei, China
}

Correspondence should be addressed to Minyou Ye; yemy@ustc.edu.cn

Received 15 April 2016; Revised 10 August 2016; Accepted 17 August 2016

Academic Editor: Arkady Serikov

Copyright (C) 2016 Chen Zhu et al. This is an open access article distributed under the Creative Commons Attribution License, which permits unrestricted use, distribution, and reproduction in any medium, provided the original work is properly cited.

An integration design platform is under development for the design of the China Fusion Engineering Test Reactor (CFETR). It mainly includes the integration physical design platform and the integration engineering design platform. The integration engineering design platform aims at performing detailed engineering design for each tokamak component (e.g., breeding blanket, divertor, and vacuum vessel). The vacuum vessel design and analysis module is a part of the integration engineering design platform. The main idea of this module is to integrate the popular CAD/CAE software to form a consistent development environment. Specifically, the software OPTIMUS provides the approach to integrate the CAD/CAE software such as CATIA and ANSYS and form a design/analysis workflow for the vacuum vessel module. This design/analysis workflow could automate the process of modeling and finite element (FE) analysis for vacuum vessel. Functions such as sensitivity analysis and optimization of geometric parameters have been provided based on the design/analysis workflow. In addition, data from the model and FE analysis could be easily exchanged among different modules by providing a unifying data structure to maintain the consistency of the global design. This paper describes the strategy and methodology of the workflow in the vacuum vessel module. An example is given as a test of the workflow and functions of the vacuum vessel module. The results indicate that the module is a feasible framework for future application.

\section{Introduction}

The China Fusion Engineering Test Reactor (CFETR) is a superconducting tokamak currently under conceptual design. The objectives of CFETR are to achieve 50 200 MW fusion power and steady-state operation with a duty cycle between 0.3 and 0.5 in order to demonstrate the feasibility of fusion energy and the self-sustainability of the fuel cycle [1].

The design work of CFETR involves many complex components such as the core plasma, breeding blanket, and divertor. Data exchange and iteration are necessary between different components as well as between physics design and engineering design. Thus, a comprehensive system code is required to perform the consistent global design of CFETR. A system code aims at self-consistent optimization of the key factor in the design of a tokamak. Several system codes have been developed by different organizations (SYCOMORE by CEA $[2,3]$, Process by CCFE $[4,5]$, and TSC by FEDC [6]).

These system codes greatly improve the efficiency of tokamak conceptual design. But they have few connections with the detailed engineering design and analysis of tokamak components. The CFETR integration design platform comprises both the physical design platform and the engineering design platform [7]. The function of the engineering platform of CFETR is to conduct the design and engineering analysis of CFETR components (e.g., magnetic coils or blanket and vacuum vessel). The required software such as ANSYS and CATIA is integrated by OPTIMUS to provide an engineering design framework. OPTIMUS is PIDO (Process Integration and Design Optimization) software. This software could integrate most kinds of CAD/CAE software, such as CATIA and ANASYS, to form a workflow for engineering design 


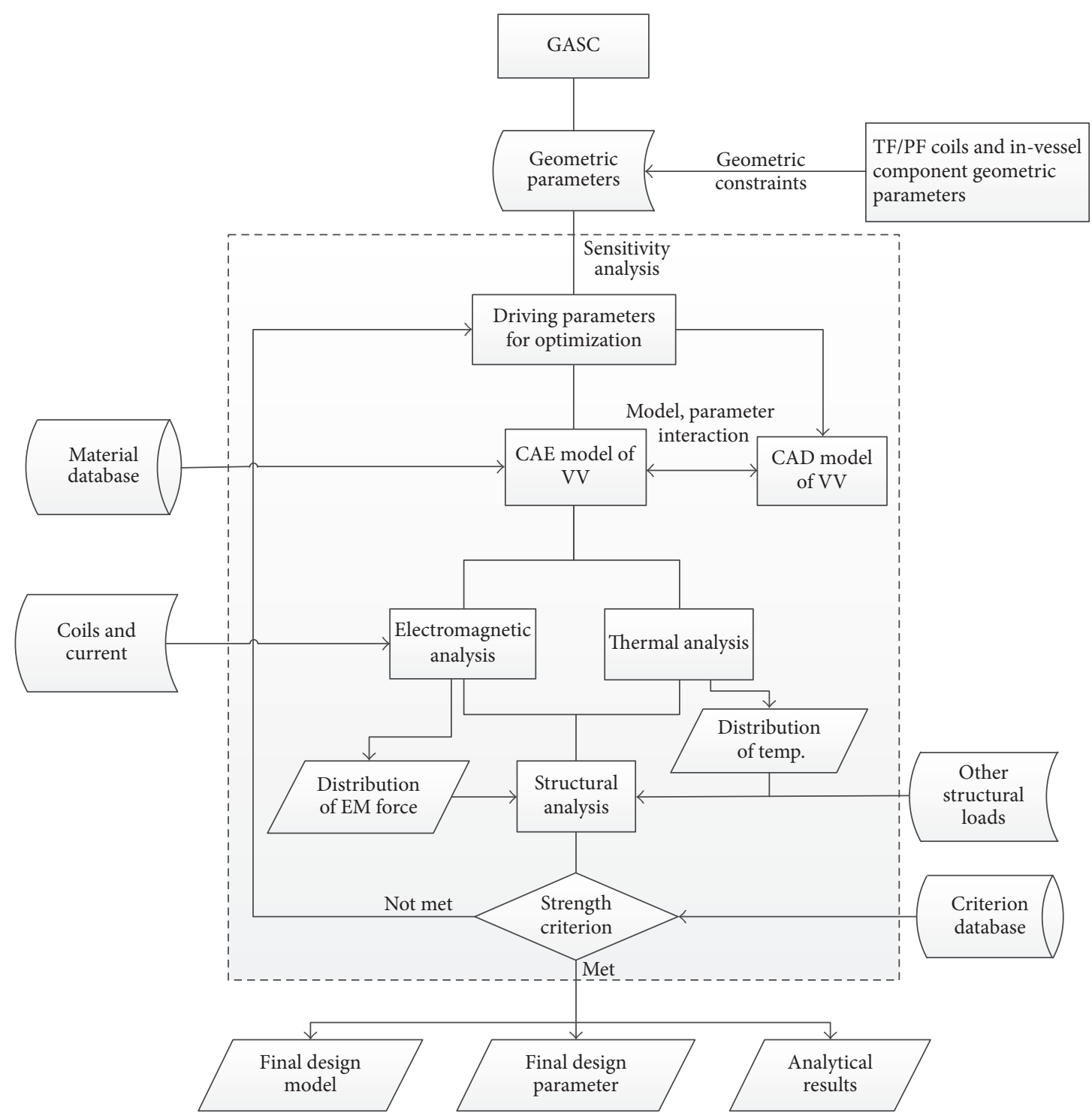

FIGURE 1: Conceptual workflow of VV module.

[8]. Every module in the engineering platform is workflow based and is independent of others. But through a standard data structure, modules can exchange data to maintain the consistency of the whole system. The input and output data are stored in a database for further utilization.

This manuscript describes the development of the engineering design and analysis module of the vacuum vessel (VV) in integration engineering platform. Section 2 is the overview of the workflow of VV module. Sections 35 present the strategy and implementation details of $\mathrm{VV}$ module. Section 6 gives an example of the application of VV module. Conclusions and prospects for future developments are outlined in Section 7.

\section{Workflow Overview}

As one of the modules in the engineering platform, the VV design and analysis module has a basic function to conduct
VV modeling and FE analysis about VV. Based on the results of FE analysis, further sensitivity analysis and optimization of geometric parameters could be done. With reference to previous experience of $\mathrm{VV}$ engineering design, the basic conceptual workflow of VV module is shown in Figure 1.

The dashed box in Figure 1 indicates the VV module. The inside of the dashed box represents the working procedure of $\mathrm{VV}$ module. The outside of the dashed box is databases and other modules that have interfaces with VV module.

Firstly, the input and output data are described as follows.

The input of VV module includes

(1) geometric parameters of VV (main input),

(2) material properties of $\mathrm{VV}$ for FE analysis,

(3) shape and current of PF/TF coils for FE analysis,

(4) structural loads (i.e., coolant pressure),

(5) structure criterion. 


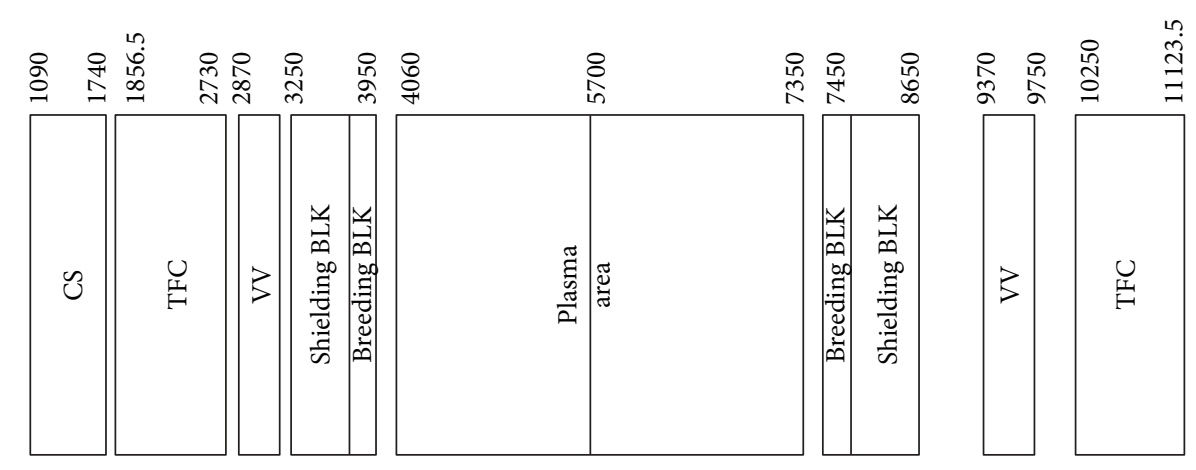

FIGURE 2: CFETR device radial space distribution.

The output of VV module includes

(1) final design model of VV,

(2) final geometric parameter of VV,

(3) results of FE analysis.

Interfaces are built into the VV module to facilitate the data exchanges of the input and output. Five interfaces have been developed to realize these exchanges:

(1) Interface between material databases

(2) Interface between assessment criterion databases

(3) Interface between working condition databases

(4) Interface between GASC [9]

(5) Interface between PF/TF modules

The data for exchanging through these interfaces are all arranged in TXT files with a predefined standard data structure. The consistency of data and model should be kept from the beginning to the end.

Generally, the procedure of a single run of the VV module is shown in Figure 1, excluding the sensitivity analysis and optimization loop. The input of single run is geometric parameters and output is the results of FE analysis. The sensitivity analysis and optimization loop are based on the single-run procedure. Steps 1-4 describe the procedure of a single run of VV module; Steps 5 and 6 describe the procedure of sensitivity analysis and optimization. More details about every step will be described in subsequent chapters accordingly.

Step 1. The VV module starts from the geometric parameters of VV, which means the model of VV should be parameterized at first to give a set of parameters that could describe the shape and structure of VV. The parameterization of CFETR VV will be discussed in the next chapter. The initial values of geometric parameters of $\mathrm{VV}$ are determined according to results of GASC (General Atomics System Code, [9]) and subject to constraints of other components (e.g., TF/PF coils and in-vessel components). GASC is a zero-dimensional system code that provides the plasma configuration and device radial space distribution (Figure 2) for every engineering module. Combining this data with the geometric parameters of TF/PF coils, the value of geometric parameters of VV could be settled to make sure the vessel is big enough to contain the plasma and small enough to fit inside the coils.

Step 2. This step involves modeling the VV in CAD and CAE software with the former geometric parameters. The parameters and the equations between these parameters are defined in both CATIA and ANSYS to achieve the parameterization of $\mathrm{VV}$, ensuring the automatic rebuilding of the model with the changing of geometric parameters afterwards. The model/parameter interaction between CAD model and CAE model means that both the model and the parameters are transferred from CAD to CAE. Instead of using the CAD model directly, the complex part of the model could be rebuilt in CAE to improve the success rate of model conversion.

Step 3. This step involves performing FE analysis based on the parameterized VV model in CAE. The FE analysis includes electromagnetic analysis, thermal analysis, and structural analysis. The results of electromagnetic and thermal analysis will be used in structural analysis to realize indirect coupling.

Step 4. This step involves outputting the model, geometric parameters, and results of $\mathrm{FE}$ analysis to the external database. The engineering platform will manage the utilization of these data in subsequent modules.

The sensitivity analysis and optimization loop are performed based on the single-run process. The steps for sensitivity analysis and optimization are as follows.

Step 5. This step consists of analyzing the sensitivity of each geometric parameter corresponding to the output of FE analysis. The sensitivity analysis could screen out the parameters with high sensitivities as the driving parameters for the subsequent optimization. The parameters with higher sensitivity have greater influence on the output, which means they should be taken into consideration in the optimization.

Step 6. In this step, the optimization loop in Figure 1 is performed. The optimization loop aims at finding the optimum value of driving geometric parameters for a specific objective. The objective of optimization could be defined according to different purposes, for example, minimizing the mass of 


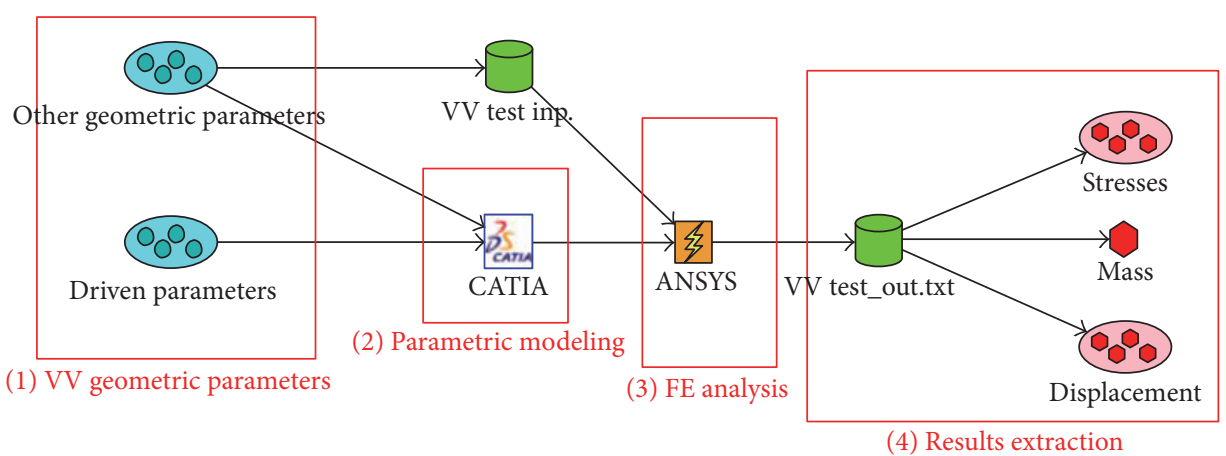

FIGURE 3: VV workflow in OPTIMUS.

$\mathrm{VV}$ in order to minimize the cost of VV. In addition, the optimizing process is subject to the constraints of a strength criterion. The results of FE analysis are checked to ensure the stress of $\mathrm{VV}$ is not exceeding the allowable stress of the material.

In order to boost the efficiency of optimization, the response surface method (RSM) and optimization algorithm (e.g., sequential linear programming, self-adaptive evolutionary programming) [10] were adopted. The response surface method requires a single-run workflow on sample points in the input space. Then, we calculate a fitting function (response surface) between input variables (geometric parameters) and the output variables (results of finite element analysis). The optimization algorithm was then applied on the response surface to find the optimum point according to the objective of optimization. Since the calculation on response surface is much faster than FE software, the combination of RSM and optimization algorithm can achieve high efficiency of optimization.

As shown in Figure 3, CATIA and ANSYS are integrated in OPTIMUS to build the framework of the VV module. Part 1 is the input of $\mathrm{VV}$ module, part 2 and part 3 are CAD modeling and FE analysis separately, and part 4 is the output of the VV module. The sensitivity analysis and optimization are all performed on this workflow.

\section{The Design of CFETR VV and Geometric Parameters}

As shown in Figure 1, the workflow begins at geometric parameters of VV. Thus, the first step is to parameterize the VV and then build a parametric model in CATIA and ANSYS in order to realize the automatic rebuilding of the model.

3.1. CFETR VV Design. The CFETR VV design is a $316 \mathrm{~L}$ SS double-wall toroidal structure. To consider the use of central space as efficiently as possible and the manufacturing difficulty, the CFETR VV has a D-shaped cross section and each D-shaped cross section is formed by one straight line and five arcs which are tangential to each other [11]. Reinforcement ribs are placed between inner shell and outer shell to ensure poloidal structural strength.

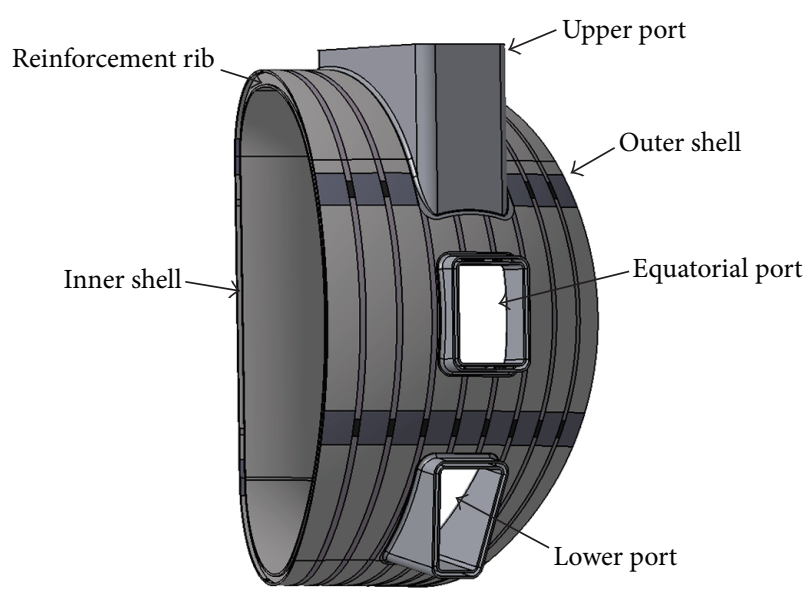

FIGURE 4: VV CAD model of CFETR.

The thickness of the inner shell and outer shell is $50 \mathrm{~mm}$ with a gap of $0.18 \mathrm{~m}$ reserved for in-wall shielding (IWS), baking, and cooling system for inboard area as well as a gap of $0.28 \mathrm{~m}$ for the outboard area at the equatorial plane. The vessel has eight vertical and eight equatorial ports for auxiliary heating, diagnostics, and remote maintenance and eight divertor ports for reassembly of divertor, utility feedthrough, and vacuum pumping. The maximum size inside the $\mathrm{VV}$ is about $6,220 \mathrm{~mm}$ in the horizontal direction and $10,270 \mathrm{~mm}$ in the vertical direction [11]. The VV is divided into 16 sectors toroidally according to the $16 \mathrm{TF}$ coils. The detailed structure of VV is shown in Figure 4.

3.2. Parameterization of $V V$. Parametric modeling offers two advantages for design: one is to facilitate the modification of the design module and the other is to promote validity and accuracy of transformation between CAD model and CAE model. Direct transformation from CAD model to CAE model mostly depends on the performance of CATIA and ANSYS, which does not have high reliability. Geometric parameters could bridge these two programs. For the complex part of the model, by using the same parameters for modeling in each program, the consistency of the model could be maintained as much as possible. 


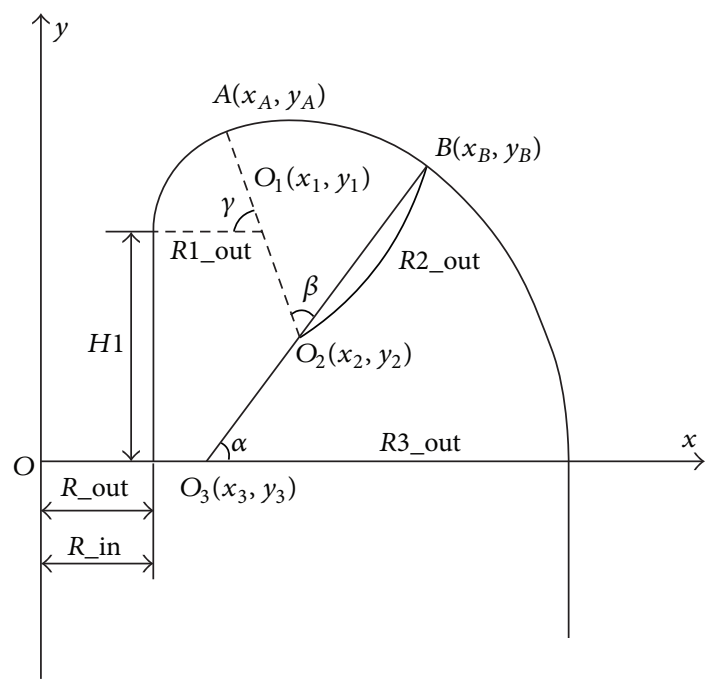

Figure 5: Geometric elements of upper half cross section of VV.

Take the parameterization of $\mathrm{D}$-shape cross section as an example. The $\mathrm{D}$-shape cross section contains one straight line and five arcs. Only the upper half of the cross section is considered since the lower half has the same structure. The detailed geometric elements of the upper half are shown in Figure 5. There are one straight line and three arcs in Figure 5, which are tangential to each other. Although there are 18 parameters to describe the geometry in Figure 5, they are not independent of each other due to the tangency among lines and arcs. The established constraint equations according to the geometry relationships are as follows:

$$
\begin{aligned}
x_{1} & =R \_ \text {out }+R 1 \_ \text {out }, \\
y_{3} & =0, \\
y_{1} & =H 1, \\
\left(x_{1}-x_{2}\right)^{2}+\left(y_{1}-y_{2}\right)^{2} & =\left(R 1 \_ \text {out }-R 2 \_ \text {out }\right)^{2}, \\
\left(x_{2}-x_{3}\right)^{2}+\left(y_{2}-y_{3}\right)^{2} & =\left(R 2 \_ \text {out }-R 3 \_ \text {out }\right)^{2}, \\
\left(x_{A}-x_{1}\right)^{2}+\left(y_{A}-y_{1}\right)^{2} & =R 1 \_ \text {out }{ }^{2}, \\
\left(x_{A}-x_{2}\right)^{2}+\left(y_{A}-y_{2}\right)^{2} & =R 2 \text { out }^{2}, \\
\left(x_{B}-x_{2}\right)^{2}+\left(y_{B}-y_{2}\right)^{2} & =R 2 \_ \text {out }^{2}, \\
\left(x_{B}-x_{3}\right)^{2}+\left(y_{B}-y_{3}\right)^{2} & =R 3 \text { out }^{2} .
\end{aligned}
$$

Then, solving these equations, the results are as follows:

$$
\begin{aligned}
& x_{2}=-b+\frac{\sqrt{b^{2}-4 a c}}{2 a}, \\
& y_{2}=\sqrt{\left(R 3 \_ \text {out }-R 2 \_ \text {out }\right)^{2}-\left(x_{2}-x_{3}\right)^{2}},
\end{aligned}
$$

where

$$
\begin{aligned}
T= & -\left(R 2 \_ \text {out }-R 1 \_ \text {out }\right)^{2}-x_{3}^{2}+x_{1}^{2} \\
& +\left(R 3 \_ \text {out }-R 2 \_ \text {out }\right)^{2}+y_{1}^{2},
\end{aligned}
$$

$$
\begin{aligned}
& S=y_{1}^{2} \times\left(R 3 \_ \text {out }-R 2 \_ \text {out }\right)^{2}, \\
& a=y_{1}^{2}+\left(x_{3}-x_{1}\right)^{2}, \\
& b=-2 \times y_{1}^{2} \times x_{3}+T \times\left(x_{3}-x_{1}\right), \\
& c=\frac{T^{2}}{4}+y_{1}^{2} \times x_{3}^{2}-S .
\end{aligned}
$$

Thus, these six parameters, H1, R_out, R1_out, $R 2$ _out, $R 3$ _out, and $x_{3}$, were one set of parameters that could completely describe the shape of the upper half of cross section consistently. In this case, they are chosen as the results of parameterization of $\mathrm{VV}$ cross section, which is shown in Figure 6. And $t 1$ represents the thickness of VV shells, $t 2$ represents the thickness of port stub extension, and $t 3$ represents the thickness of reinforcement ribs. Along with parameterization of $\mathrm{D}$-shape cross section, the position and cross section of the port and other parts of VV were parameterized using the same method. The geometric parameters of port cross sections are shown in Figure 7.

The detailed design parameters were shown in Table 1. The parameters from $H 1$ to $x_{3}$ in describe geometry of $\mathrm{D}$ shape cross section of inner and outer shell. The parameters from wd $h$ to ang low describe the position and shape of every port and the parameters from $t 1$ to $t 4$ represent the thickness of every part of VV as shown in Figures 6 and 7. The parameter secn represents the number of $\mathrm{VV}$ sectors and ang represents the angle between reinforcement ribs.

Along with these parameters, the constraint equations have been established in both CATIA and ANSYS. When one parameter changes, the model automatically changes and keeps smooth joins everywhere. But the allowed value range of each parameter was restricted to make sure the vessel is big enough to contain the plasma and small enough to fit inside the coils.

\section{FE Analytic Process of VV}

ANSYS v15.0 was adopted for performing the FE analyses of $\mathrm{VV}$, which include electromagnetic field analysis, thermal analysis, and structural analysis. With the same FE model, these analyses could be coupled indirectly. The EM force distribution and temperature distribution are, respectively, read from result files from electromagnetic analysis and thermal analysis.

4.1. Type of Loads and Working Conditions. The mechanical loads acting on the VV can be divided into four independent categories.

(1) Electromagnetic (EM) Loads. The magnetic field for the confinement of plasma is generated by the current in the superconducting coils, and the plasma current itself, when the plasma is disrupting, the magnetic flux going through the VV will change which induces current in the structure of VV module. The induced current interacting with the magnetic field will generate EM forces as additional loads on VV 


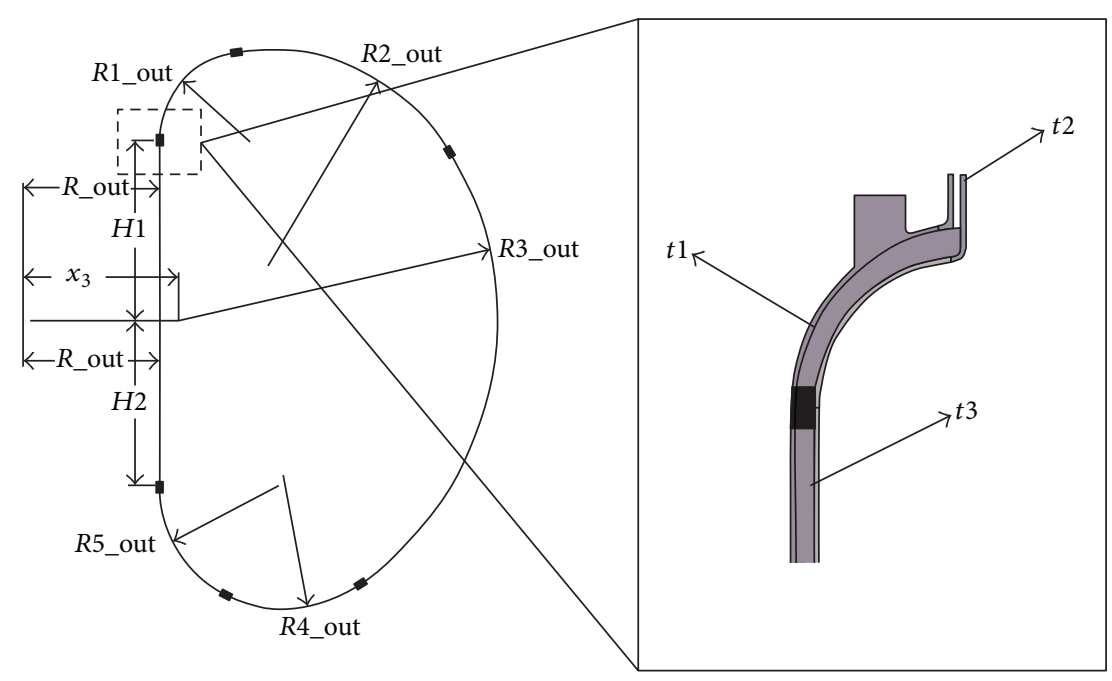

FIGURE 6: Main geometric parameters of VV.

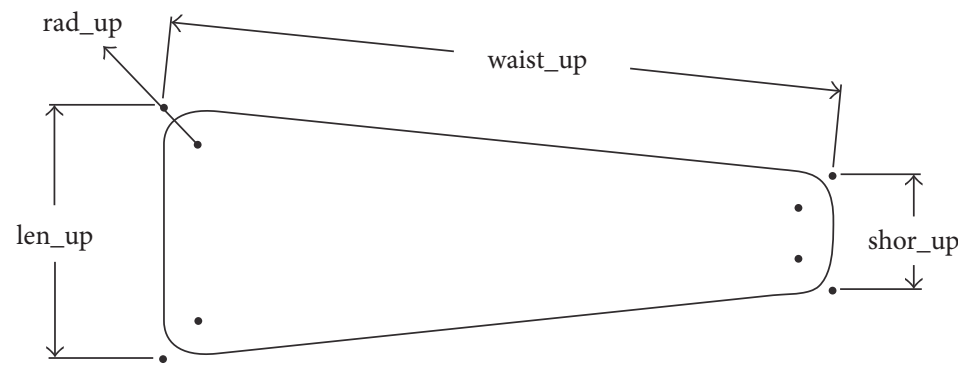

(a)

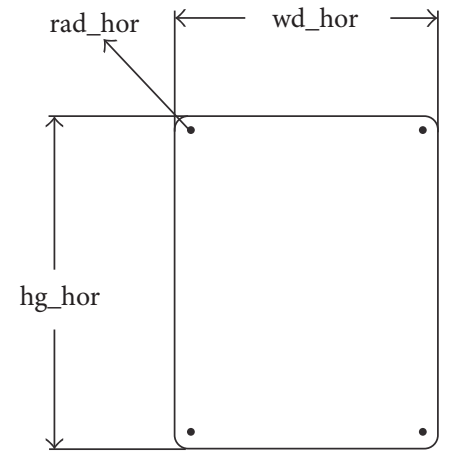

(b)

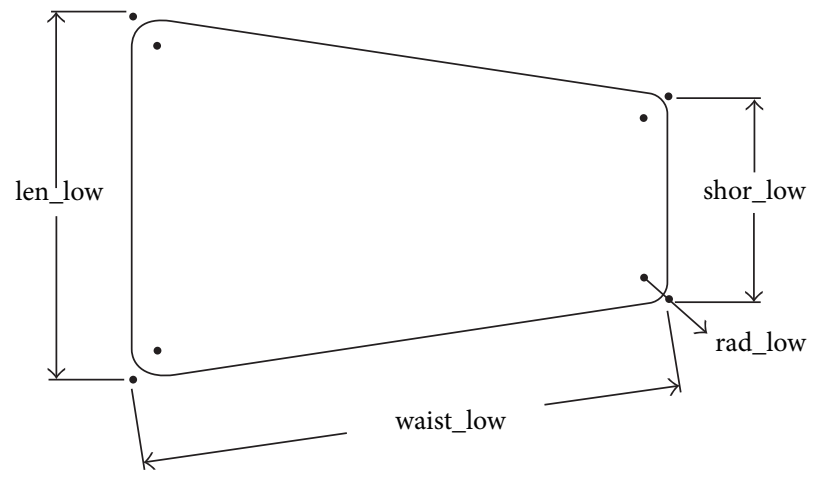

(c)

FIGURE 7: Geometric parameters of port cross sections. (a) Cross section of the upper port; (b) cross section of the equatorial port; (c) cross section of the lower port.

module [12] including plasma major disruption (MD) event, vertical displacement event (VDE), and Halo current event.

(2) Thermal Loads. Temperature gradients inside the VV structure are caused by nuclear heating on one side and water cooling on the other side, including baking heat, nuclear heat during plasma normal operation.

(3) Pressure Loads. These include coolant pressure and incidental VV internal and external pressure.
(4) Inertial Loads. These are caused by acceleration due to gravity and seismic events.

Taking all the mechanical loads into consideration is the objective of VV module but only loads listed in Table 2 have been considered in the FE analysis.

4.2. Extraction of Results. The results of FE analysis are used to examine the structural strength of VV. Stresses are 


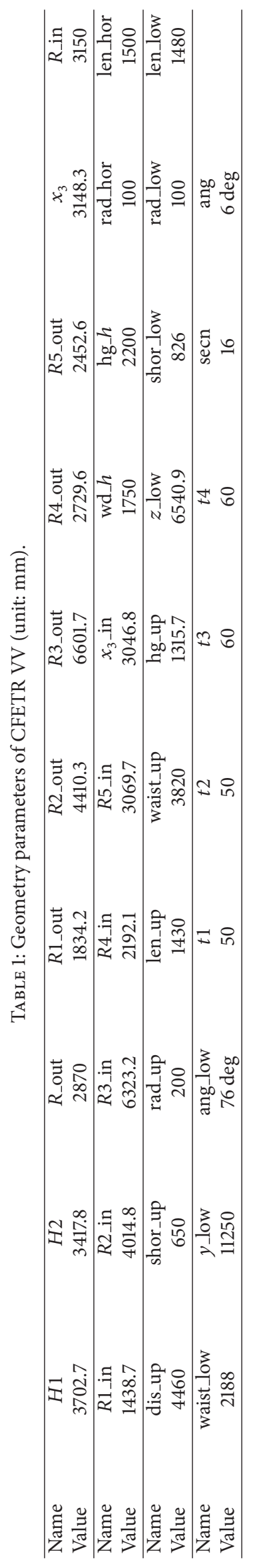


TABLE 2: Types of loads performed in VV module.

\begin{tabular}{lc}
\hline Type of load & Type of analysis \\
\hline EM forces from MD event & Electromagnetic analysis \\
Nuclear heat during normal operation & Thermal analysis \\
Coolant pressure & Structural analysis \\
Gravity (dead weight) & Structural analysis \\
\hline
\end{tabular}

TABLE 3: Design criterion of mechanical strength.

\begin{tabular}{|c|c|c|c|c|}
\hline & $P_{m}(\mathrm{MPa})$ & $P_{l}(\mathrm{MPa})$ & $P_{m}\left(P_{l}\right)+P_{b}(\mathrm{MPa})$ & $P_{l}+P_{b}+Q(\mathrm{MPa})$ \\
\hline Temp. & $S_{m}$ & $1.5 S_{m}$ & $1.5 S_{m}$ & $3 S_{m}$ \\
\hline $20^{\circ} \mathrm{C}$ & 147 & 221 & 221 & 441 \\
\hline $100^{\circ} \mathrm{C}$ & 147 & 221 & 221 & 441 \\
\hline $200^{\circ} \mathrm{C}$ & 130 & 195 & 195 & 520 \\
\hline
\end{tabular}

extracted and classified into four types according to ASMEVIII [13].

(1) General Primary Membrane Stress $\left(P_{m}\right)$ is stress of uniform distribution along the direction of the thickness of the vacuum vessel.

(2) Local Primary Membrane Stress $\left(P_{l}\right)$ is membrane stress only on local area of VV.

(3) Primary Bending Stress $\left(P_{b}\right)$ is stress of linear distribution along the direction of the thickness of the vacuum vessel.

(4) Secondary Stress (Q) mainly comprises thermal stress.

Referring to ASME-VIII-2 and ITER VV design criterion $[13,14]$, the fourth strength theory and von Mises are used to evaluate the strength of VV. Table 3 shows the allowable stress under different temperatures. $S_{m}$ represents allowable stress for $316 \mathrm{~L}$.

\section{Sensitivity Analysis and Optimization}

Generally, there are dozens of parameters to describe a VV (Table 1). Some of them do have important influence on the strength of VV, while others do not. Optimizing all of them is a time-consuming and meaningless work. Those who have significant influence on VV mechanical strength are considered as driven parameters in optimization. Others stay fixed during optimization. The objective of optimization is to find the optimal value of these driven parameters. Sensitivity analysis is the method to screen them from all the geometric parameters.

5.1. Sensitivity Analysis. Sensitivity analysis is the study of how the uncertainty in the output of a mathematical model or system (numerical or otherwise) can be apportioned to different sources of uncertainty in its inputs [15].

A variance approach to calculate global sensitivity index (Sobol index) based on response surface method is provided in VV module. Response surfaces are also known as meta models, surrogates, emulators, auxiliary models, and so forth. The main idea of RSM is to use a sequence of designed
TABLE 4: Results of sensitivity analysis.

\begin{tabular}{lccc}
\hline \multirow{2}{*}{ Parameter } & \multicolumn{3}{c}{$\begin{array}{c}\text { Impact on FE results } \\
\text { (delta stress/MPa) }\end{array}$} \\
\hline$t 1(50 \pm 20 \mathrm{~mm})$ & I & II & III \\
$t 2(50 \pm 20 \mathrm{~mm})$ & 5.7 & 39.9 & 54.2 \\
$t 3(50 \pm 20 \mathrm{~mm})$ & 2.2 & 37.2 & 38.7 \\
$H 1(3702.7 \pm 100 \mathrm{~mm})$ & 0.1 & 6.4 & 2.3 \\
$H 2(3417.8 \pm 100 \mathrm{~mm})$ & 0.3 & 11.6 & 5.1 \\
$R 1$ out $(1834.2 \pm 100 \mathrm{~mm})$ & 0.5 & 12.8 & 14.7 \\
$R 2$ out $(4410.3 \pm 100 \mathrm{~mm})$ & 0.1 & 14.5 & 13.5 \\
$R$ 3_out $(6601.7 \pm 100 \mathrm{~mm})$ & 2.8 & 9.4 & 10.4 \\
$R 4$ out $(2729.6 \pm 100 \mathrm{~mm})$ & 0.3 & 3.5 & 3.4 \\
$R 5$ out $(2452.6 \pm 100 \mathrm{~mm})$ & 0.2 & 5.3 & 4.8 \\
\hline
\end{tabular}

Note. I: stress caused by gravity and coolant pressure; II: stress caused by gravity and nuclear heat; III: stress caused by gravity, coolant pressure, EM force, and nuclear heat.

experiments to obtain an optimal response, which is easy to estimate and apply. OPTIMUS has a wide range of methods in building RSM models, including least square method based on Taylor expansion and user-defined expansion, interpolation method based on Krigin method, and Radial Basis Function (RBF) method $[10,16,17]$. According to the RSM model, the program can calculate Sobol index of each input to decide their contribution to the outputs.

Local sensitivity analysis is also particularly useful when screening high dimensional models. One at a time (OAT) is a choice. The principle of OAT is simple: each parameter is varied successively from a given nominal value while keeping the other parameters constant. Repeating this procedure a limited number of times for each parameter, one can obtain a rough idea of its effect on output parameters [18].

5.2. Optimization. Local optimization search algorithm and global optimization search algorithm both are available in the module. The former includes sequential linear programming method and sequential square programming method. The latter includes self-adaptive evolutionary programming method (SAE) and differential evolutionary programming method [19-21]. Combined with RSM, these methods could finish optimization within a short time, since response surface method is used instead of repetitive FE analysis. The task of optimization can be more effective and convenient. Besides, the utility of global optimization algorithms makes it more likely to achieve globally optimal solution.

After all, the response surface method is only an approximation to FE analysis; it cannot completely replace the FE analysis. The results of the optimization need to be verified by FE analysis. Combining this method with FE analysis, the global optimization could be realized with high efficiency.

\section{Testing of VV Module}

In order to test the function of VV module, an example was performed. Table 4 shows the results of sensitivity analysis based on OAT method. By performing single-run process 
TABLE 5: Results comparison of RSM and FE analysis.

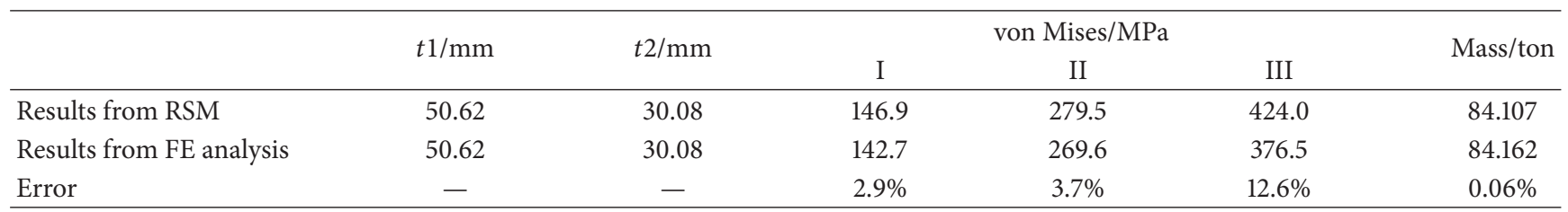

Note. I: stress caused by gravity and coolant pressure; II: stress caused by gravity and nuclear heat; III: stress caused by gravity, coolant pressure, EM force, and nuclear heat.

TABLE 6: Configurations of hardware.

\begin{tabular}{lc}
\hline Hardware & Model \\
\hline CPU & Xeon E5-2680v3 2.50 GHz $\times 2$ \\
Memory & Samsung DDR4 2133 MHz $(64 \mathrm{~GB})$ \\
GPU & Nvidia Quadro K6000 (with GPU acceleration) \\
\hline
\end{tabular}

with the change of input, the impact of each input parameter on results of FE analysis indicates their sensitivity. The effect of each input variable is converted to a stress increment (delta stress). The impact on FE results of $t 1$ and $t 2$ is at least double that of other parameters, which indicates that the stress level of VV greatly depends on $t 1$ and $t 2$. Thus, these two parameters were chosen as the driven parameters in optimization shown in Table 4.

Then, optimization was performed to get the optimum value of $t 1$ and $t 2$. The constraints of stress are set as in Table 3 . The objective of optimization is to minimize the mass of VV (assuming that cost is proportional to mass). With the Latinhypercube sampling, the single-run process was performed at different experimental points in the parameter space formed by $t 1$ and $t 2$. Then, RSM was established based on these results of single-run process. At last, SAE was adopted on RSM model to find the optimum point.

Table 5 shows the optimal values of $t 1$ and $t 2$, which are $50.62 \mathrm{~mm}$ and $30.08 \mathrm{~mm}$, respectively, while stress levels were within the design criterion. Then, a single FE analysis was performed with optimal values of $t 1$ and $t 2$ to give a comparison of results from RSM and from FE analysis (Table 5). Most errors between RSM and FE analysis are under $5 \%$ but some of them are over $10 \%$. Thus, improving the quality of RSM is one of the future tasks in order to get more reasonable optimization results.

The function of VV module was tested by using a workstation with a configuration of the hardware as shown in Table 6. But the whole integration platform is going to be deployed on servers in the future plan. The calculation time changes a lot due to different configurations.

The average calculation time of individual FE analysis is about 6 hours and 48 minutes. According to the SAE (selfadaptive evolutionary) method, optimization without RSM usually needs to perform FE analysis 100 to 300 times. Thus, the calculation time is at least 600 hours. But, with RSM method, the results are calculated through response surface instead of FE software. The calculation based on response surface is very fast. It takes less than 5 seconds to accomplish 100 to 300 calculations on response surface. But building the response surface still needs a couple of calculations on FE software. In this case, the FE analysis is performed 24 times to build the response surface. And 186 times of calculations were performed on response surface for the optimization. The calculation time of the overall procedure of optimization with RSM is 163 hours and 13 minutes. Without RSM, the same 186 calculations should have been performed on FE software, which will take nearly 1264 hours and 48 minutes. Even if the number of calculations is more or less different on FE software, the calculation time is still more than 600 hours. Therefore, with the help of RSM, the calculation can achieve a higher efficiency.

\section{Conclusion and Perspective}

The VV design and analysis module of CFETR engineering design platform provides a new approach to conduct engineering design and parameter optimization of the vacuum vessel. The integration of different software made it possible to allow automation and analysis. Combined with RSM, the module gives a more efficient way for engineers to realize the global geometric optimization.

The integration design platform is still under development. Currently, the basic framework has been established. The interfaces and iteration between modules are still planned for future development. For vacuum vessel module, in the next work plan, new analyses such as seismic analysis and buckling analysis should be added to this module and the RSM also should be improved to achieve a higher accuracy.

\section{Competing Interests}

The authors declare that they have no competing interests.

\section{Acknowledgments}

This work is supported by the National Magnetic Confinement Fusion Research Program of China (Grants nos. 2014GB110001 and 2014GB110002). The authors would like to express their thanks to Vincent Chen, Xuebing Peng, Zhengping Luo, Yong Guo, Nan Shi, and Kun Xu from the CFETR integration platform team.

\section{References}

[1] Y. Wan, "Mission of CFETR," in Proceedings of the ITER Training Forum \& 2nd Workshop on MFE Development Strategy, pp. 1-6, 2012. 
[2] G. Falchetto, D. Coster, R. Coelho et al., "The European Integrated Tokamak Modelling (ITM) effort: achievements and first physics results," Nuclear Fusion, vol. 54, no. 4, Article ID 043018, 2014.

[3] C. Reux, L. Di Gallo, F. Imbeaux et al., "DEMO reactor design using the new modular system code SYCOMORE," Nuclear Fusion, vol. 55, no. 7, Article ID 073011, 2015.

[4] M. Kovari, R. Kemp, H. Lux, P. Knight, J. Morris, and D. J. Ward, "PROCESS: a systems code for fusion power plants-part 1: physics," Fusion Engineering and Design, vol. 89, no. 12, pp. 3054-3069, 2014.

[5] P. J. Knight, A User Guide to the PROCESS Systems Code, CCFE, 2013.

[6] R. L. Reid, R. J. Barrett, T. G. Brown et al., Tokamak Systems Code, ORNL/FEDC-84/9, Oak Ridge National Laboratory, Oak Ridge, Tenn, USA, 1985.

[7] M. Y. Ye, S. J. Wang, Z. W. Wang et al., "Development of system code of CFETR design," in Proceedings of the IEEE 26th Symposium on Fusion Engineering (SOFE '15), pp. 1-4, Austin, Tex, USA, May 2015.

[8] Noesis Solution NV, Optimus Rev. 10.13 Theory Manual, Noesis Solutions, Leuven, Belgium, 2012.

[9] V. S. Chan, A. E. Costley, B. N. Wan, A. M. Garofalo, and J. A. Leuer, "Evaluation of CFETR as a fusion nuclear science facility using multiple system codes," Nuclear Fusion, vol. 55, no. 2, Article ID 023017, 2015.

[10] J. P. Kleijnen, "Kriging metamodeling in simulation: a review," European Journal of Operational Research, vol. 192, no. 3, pp. 707-716, 2009.

[11] Y. T. Song, S. T. Wu, J. G. Li et al., "Concept design of CFETR tokamak machine," IEEE Transactions on Plasma Science, vol. 42, no. 3, pp. 503-509, 2014.

[12] S. Liu, M. Chen, M. Lei, M. Lu, and Z. Wang, "Electromagnetic and structural analysis on vacuum vessel for CFETR during plasma major disruption," Journal of Fusion Energy, vol. 33, no. 6, pp. 713-719, 2014.

[13] American Society of Mechanical Engineers (ASME), Boiler and Pressure Vessel Code Section III, American Society of Mechanical Engineers, New York, NY, USA, 2007.

[14] ITER Organization, Design DescriptionDocument (DDD) for the ITER Vacuum Vessel (VV), ITER_D_22FPWQ v4.0, 2012.

[15] A. Saltelli, M. Ratto, T. Andres et al., Global Sensitivity Analysis: The Primer, John Wiley \& Sons, New York, NY, USA, 2008.

[16] D. G. Cacuci and M. Ionescu-Bujor, "A comparative review of sensitivity and uncertainty analysis of large-scale systems-II: statistical methods," Nuclear Science and Engineering, vol. 147, no. 3, pp. 204-217, 2004.

[17] I. M. Sobol', "On sensitivity estimation for nonlinear mathematical models," Matematicheskoe Modelirovanie, vol. 2, no. 1, pp. 112-118, 1990.

[18] A. Saltelli, S. Tarantola, F. Campolongo, and M. Ratto, Sensitivity Analysis in Practice: A Guide to Assessing Scientific Models, John Wiley \& Sons, Chichester, UK, 2004.

[19] R. T. Haftka and Z. Gürdal, Elements of Structural Optimization, vol. 11, Springer, 2012.

[20] R. Horst and H. Tuy, Global Optimization: Deterministic Approaches, Springer Science \& Business Media, 2013.

[21] R. Storn and K. Price, "Differential evolution-a simple and efficient heuristic for global optimization over continuous spaces," Journal of Global Optimization, vol. 11, no. 4, pp. 341359, 1997. 

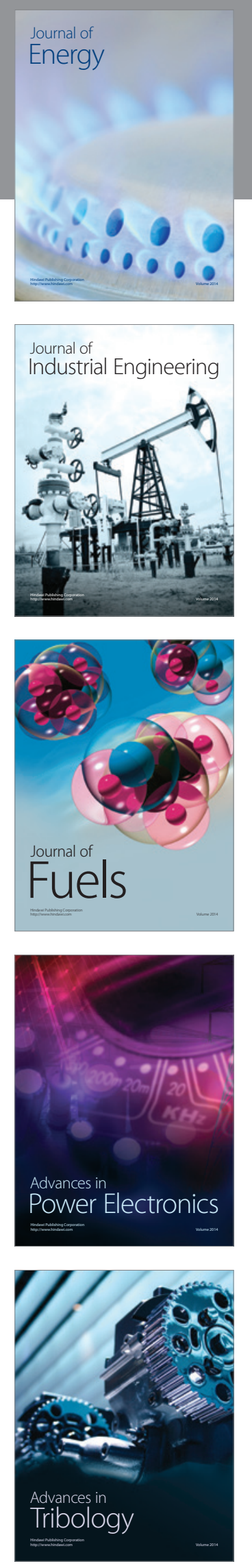
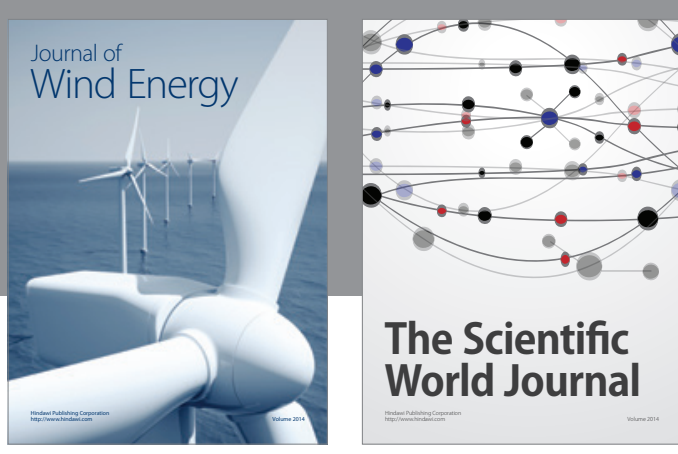

The Scientific World Journal
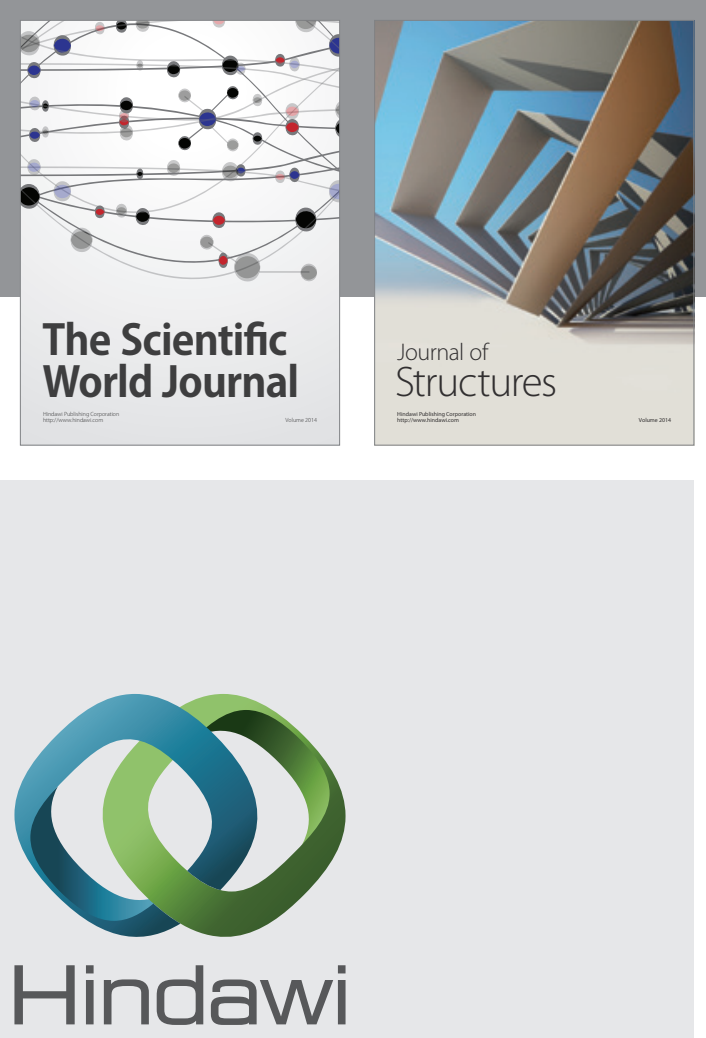

Submit your manuscripts at

http://www.hindawi.com
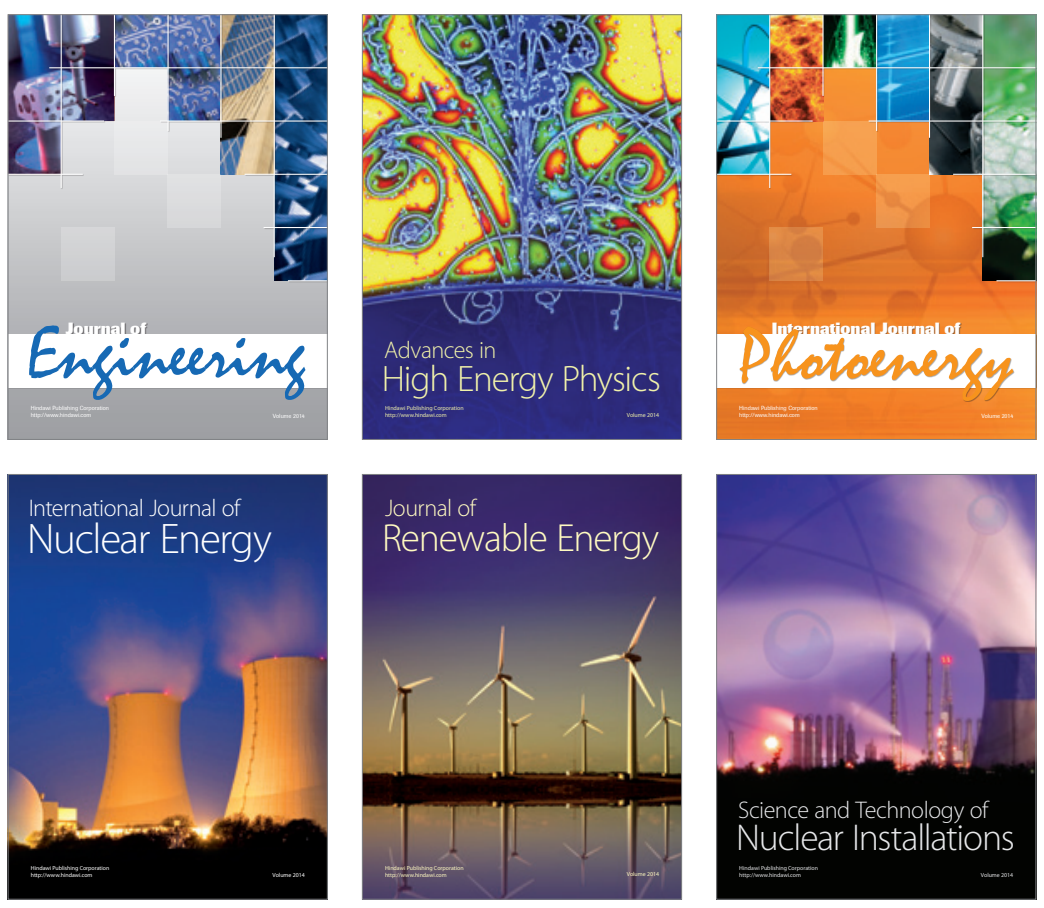
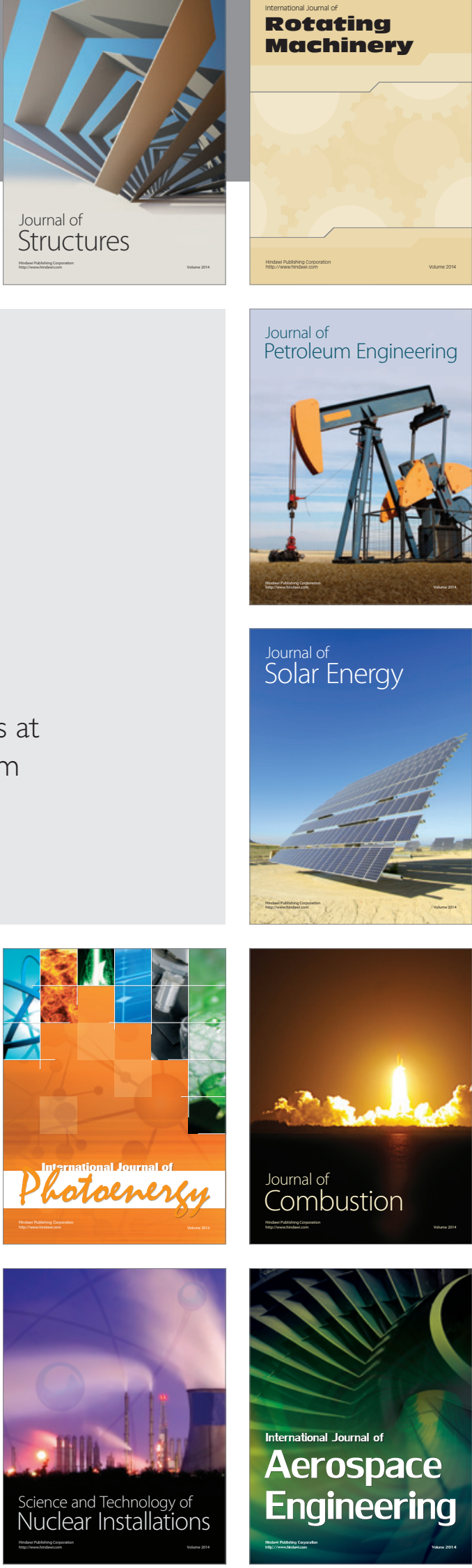